Claret Medical, GLG Consulting, Guidepoint Global, Imperative Care, Medtronic, Rebound, StimMed. 4; C; NeXtGen Biologics, RAPID Medical, Claret Medical, Cognition Medical, Imperative Care (formerly the Stroke Project), Rebound Therapeutics, StimMed, Three Rivers Medical.

\section{E-066 STRUCTURED REPORTING OF ANEURYSM RUPTURE RISK UTILIZING GEOMETRIC AND HEMODYNAMIC ANALYSIS (STARR) - A SOPHISTICATED APPROACH TOWARDS PATIENT COUNSELING}

${ }^{1} \mathrm{D}$ Behme*, ${ }^{2}$ Reinitz, ${ }^{3} \mathrm{O}$ Beuing, ${ }^{1} \mathrm{M}$ Thormann, ${ }^{4} \mathrm{~B}$ Neyazi, ${ }^{4} \mathrm{E}$ Sandalcioglu, ${ }^{1} \mathrm{~A}$ Mpotsaris, ${ }^{5} B$ Preim, ${ }^{6}$ P Berg, ${ }^{7} S$ Saalfeld. ${ }^{1}$ Neuroradiology, University Clinic Magdeburg, Magdeburg, Germany; ${ }^{2}$ Research Campus Stimulate, University Magdeburg, Magdeburg, Germany; ${ }^{3}$ Neuroradiology, AMEOS Hospital Bernburg, Bernburg, Germany; ${ }^{4}$ Neurosurgery, University Clinic Magdeburg, Magdeburg, Germany; ${ }^{5}$ Department of Simulation and Graphics, University Magdeburg, Magdeburg, Germany; ${ }^{6}$ Research Campus Stimulate; Department of Fluid Mechanics and Technical Flows, University Magdeburg, Magdeburg, Germany; ${ }^{7}$ Research Campus Stimulate; Department of Simulation and Graphics, University Magdeburg, Magdeburg, Germany

\subsection{6/neurintsurg-2021-SNIS.161}

Introduction/Purpose Counseling of patients harboring unruptured intracranial aneurysms (UIAs) has remained challenging despite the availability of several risk scores assessing the aneurysm rupture risk for individual patients. However, besides aneurysm size and simple morphological features no advanced hemodynamic or geometric factors have been used in every patient counselling so far, although recent research has identified several of these factors to be associated with aneurysm rupture risk. The structured report of several geometric and hemodynamic aneurysm features was developed to amend counseling of patients with UIAs in everyday clinical practice.

Material and Methods Based on current state-of-the-art and experiences from daily clinical practice a selection of morphological and hemodynamical parameters (5 parameters each, figure 1) that have been associated with aneurysm rupture risk was generated. Next, a structured report was designed such that these parameters are presented in an intuitive and clear manner. Color legends from red to green are used to visualize patient specific rupture risk. Special care was taken to account for non-uniform parameter distributions. The design study was evaluated with a clinical expert. For the extraction of these parameters customized software tools were used that can be carried out remotely. The patient image data is semi-automatically processed yielding the structured report.

Results We developed a semi-automatic workflow to incorporate current state-of-the-art aneurysm rupture risk analysis into a structured report such that the information is available in the daily clinical use without dedicated hardware or engineers required at the hospital. All listed parameters were extracted based on recent literature.

Conclusion Structured reporting of geometric and hemodynamic analysis of IAs is feasible and can be combined for an aneurysm specific rupture risk stratification amending a more sophisticated tool to patient counseling harboring UIAs. Figure legend: Illustration of the structured report comprising patient specific information (e.g. age, sex,...), the PHASES and UIATS scores as well as five different views showing the shape of the aneurysm from different angles as well as the aneurysm and its parent artery. Five morphological parameters are combined, as well seven hemodynamical parameters. All parameters are
Aneurysma Rupture Risk Assesment

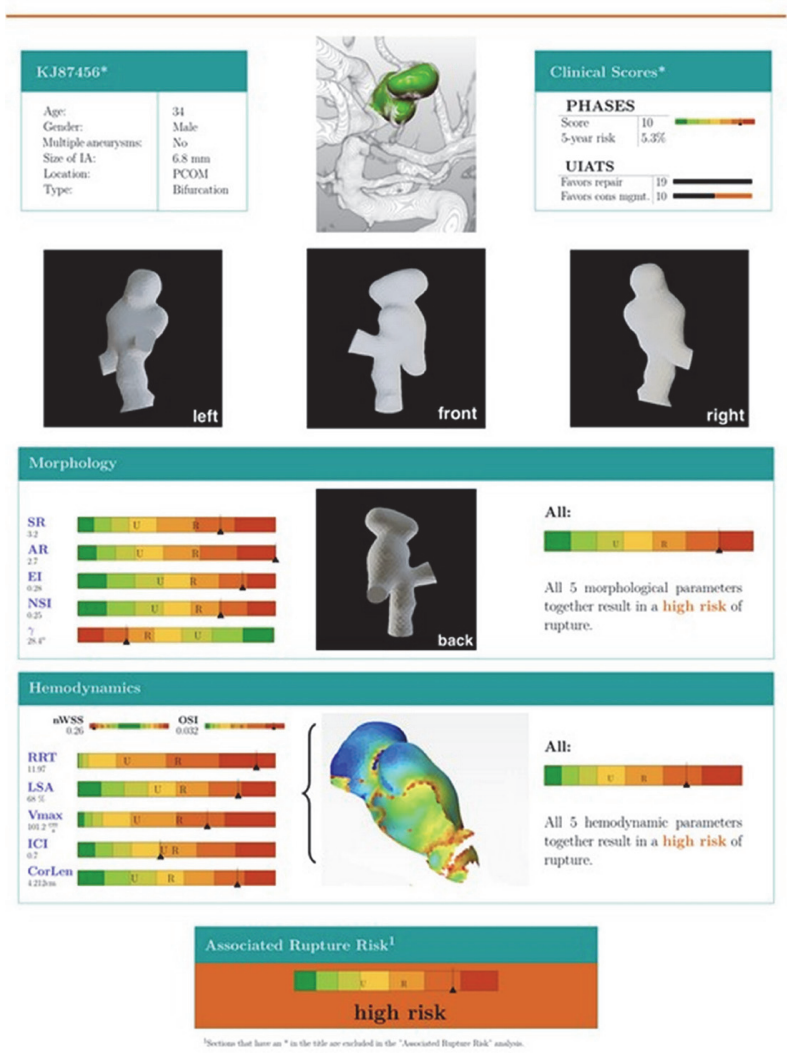

Abstract E-066 Figure 1

presented in a structured manner. Tool tips allow for explanation for the clinical expert as well as the patients.

Disclosures D. Behme: None. I. Reinitz: None. O. Beuing: None. M. Thormann: None. B. Neyazi: None. E. Sandalcioglu: None. A. Mpotsaris: None. B. Preim: None. P. Berg: None. S. Saalfeld: None.

\section{E-067 IMPORTANCE OF FIRST PASS REPERFUSION IN ENDOVASCULAR STROKE CARE - INSIGHTS FROM STAR}

${ }^{1}$ I Maier, ${ }^{2} \mathrm{M}$ Psychogios, ${ }^{1} \mathrm{~J}$ Liman, ${ }^{3} \mathrm{~S}$ Al Kasab, ${ }^{4} \mathrm{E}$ Almallouhi, ${ }^{5} \mathrm{~A}$ Alawieh, ${ }^{6} \mathrm{~S}$ Wolfe, ${ }^{6} \mathrm{~K}$ Fargen, ${ }^{7} \mathrm{~A}$ Arthur, ${ }^{8} \mathrm{~N}$ Goyal, ${ }^{9} \mathrm{~T}$ Dumont, ${ }^{10} \mathrm{P}$ Kan, ${ }^{11} \mathrm{~J}$ Kin, ${ }^{12} \mathrm{R}$ De Leacy, ${ }^{13} \mathrm{~J}$ Osbun, ${ }^{14} \mathrm{~A}$ Rai, ${ }^{15} \mathrm{P}$ Jabbour, ${ }^{16} \mathrm{~J}$ Grossberg, ${ }^{17} \mathrm{M}$ Park, ${ }^{18} \mathrm{R}$ Starke, ${ }^{19} \mathrm{R}$ Crosa, ${ }^{4} \mathrm{~A}$ Spiotta, ${ }^{20} \mathrm{D}$ Behme*. ${ }^{1}$ Neurology, University Clinic Göttingen, Göttingen, Germany: ${ }^{2}$ Neuroradiology, University Basel, Basel, Switzerland; ${ }^{3}$ Neurology and Neurosurgery, Medical University of South Carolina, Charleston, SC; ${ }^{4}$ Neurosurgery, Medical University of South Carolina, Charleston, SC; ${ }^{5}$ Neurosurgery, Emory University School of Medicine, Atlanta, GA; ${ }^{6}$ Neurosurgery, Wake Forest School of Medicine, Winstom Salem, NC; ${ }^{7}$ Neurosurgery, University of Tennessee Health Science Center, Memphis, TN; ${ }^{8}$ Neurology, University of Tennessee Health Science Center, Memphis, TN; ${ }^{9}$ Neurosurgery, University of Arizona Health Science, Tuscon, AZ; ${ }^{10}$ Neurosurgery, Baylor School of medicine, Houston, $T X_{i}{ }^{11}$ Neurology, Chonnam National University, Seoul, Korea, Democratic People's Republic of; ${ }^{12}$ Neurosurgery, Mount Sinai Health System, New York, NY; ${ }^{13}$ Neurosurgery, Washington University School of Medicine, St. Louis, MO; ${ }^{14}$ Radiology, West Virginia School of Medicine, Morgantown, WV; ${ }^{15}$ Neurosurgery, Thomas Jefferson University Hospital, Philadelphia, PA; ${ }^{16}$ Neurosurgery, Emory University School of Medicine, Charleston, SC: ${ }^{17}$ Neurosurgery, University of West Virginia, Charlottesville, $W V ;{ }^{18}$ Neurosurgery, University of Miami Health System, Miami, FL; ${ }^{19}$ Neurosurgery, Endovascular Neurological Center, Montevideo, Uruguay; ${ }^{20}$ Neuroradiology, University Clinic Magdeburg, Magdeburg, Germany

10.1136/neurintsurg-2021-SNIS.162 
Background Endovascular therapy (EVT) has become the first line treatment strategy for patients with large vessel occlusion strokes (LVOS). However, often more than one thrombectomy maneuver - either using stent-retrievers, thrombus aspiration or combined approaches - is necessary to achieve reperfusion. A true first pass effect with improved functional outcomes after MT has been described previously in single center data. Aim of the present study is to investigate the effect of true first-pass complete recanalization vs. multi-pass complete recanalization on functional outcome in a large, multicenter stroke database.

Methods Patients who underwent MT for LVOS in the anterior cerebral circulation between January 2014 to January 2021 and achieved complete reperfusion were identified from the Stroke Thrombectomy and Aneurysm Registry (STAR). We compared functional outcomes of patients with first- (defined as mTICI 3 after a single thrombectomy maneuver) vs. multiple-pass complete reperfusion (defined as mTICI 3 after $\geq 1$ thrombectomy maneuvers).

Results A total of 1481 patients with anterior circulation LVOS and successful recanalization were included in the analysis. First-pass complete recanalization was achieved in 778 patients vs. 703 patients with multi-pass complete reperfusion. Patients with first pass complete recanalization had lower NIHSS at baseline $(15 \pm 7$ vs $16 \pm 7$, $\mathrm{p}=0.056)$, had higher ASPECTS at baseline $(8.3$ vs 7.8 , $\mathrm{p}<0.001)$, were less likely to be male $(47 \%$ vs $51 \%$, $\mathrm{p}=0.078)$ and to have intracranial ICA occlusions $(14 \%$ vs. $27 \%)$ as well as more likely to have M1/M2 occlusions (86\% vs. $73 \%, \mathrm{o}<0.001)$, diabetes mellitus $(28 \%$ vs. $24 \%$, $\mathrm{p}=0.076)$, atrial fibrillation $(37 \%$ vs. $32 \%, \mathrm{p}=0.064)$. Patients with first pass complete reperfusion hat a trend towards a lower discharge NIHSS (8 vs. 10, $\mathrm{p}=0.075$ ) and higher improvement of the NIHSS (7 vs. 5, p=0.168). Among the factors lower age (OR 0.966, p<0.010), lower pre-stroke mRS (OR 0.601, p<0.001), diabetes mellitus (OR 0.612, $\mathrm{p}=0.014$ ), higher ASPECTS (OR 1.183, $\mathrm{p}<0.001)$ first pass complete recanalization persisted as a independent predictor of favorable functional outcome (defined as modified Rankin Scale $\leq 2$; OR 1.49, p=0.026).

Conclusion This analysis of a large, multi-center stroke database confirms the importance of first pass reperfusion in endovascular stroke care.

Disclosures I. Maier: None. M. Psychogios: None. J. Liman: None. S. Al Kasab: None. E. Almallouhi: None. A. Alawieh: None. S. Wolfe: None. K. Fargen: None. A. Arthur: None. N. Goyal: None. T. Dumont: None. P. Kan: None. J. Kin: None. R. De Leacy: None. J. Osbun: None. A. Rai: None. P. Jabbour: None. J. Grossberg: None. M. Park: None. R. Starke: None. R. Crosa: None. A. Spiotta: None. D. Behme: None.

\section{E-068 TOWARDS AUTOMATED INTRACRANIAL ANEURYSMAL BLEB DETECTION}

${ }^{1} \mathrm{D}$ Behme*, ${ }^{2} \mathrm{~L}$ Langolf, ${ }^{1} \mathrm{M}$ Thormann, ${ }^{1} \mathrm{~A}$ Mpotsaris, ${ }^{3} \mathrm{~B}$ Preim, ${ }^{4} \mathrm{P}$ Berg, ${ }^{5} \mathrm{~S}$ Saalfeld. ${ }^{1}$ Neuroradiology, University Clinic Magdeburg, Magdeburg, Germany; ${ }^{2}$ Research Campus Stimulate, University Magdeburg, Magdeburg, Germany; ${ }^{3}$ Department of Simulation and Graphics, University Magdeburg, Magdeburg, Germany; ${ }^{4}$ Research Campus Stimulate; Department of Fluid Mechanics and Technical Flows, University Magdeburg, Magdeburg, Germany; ${ }^{5}$ Research Campus Stimulate; Department of Simulation and Graphics, University Magdeburg, Magdeburg, Germany

\subsection{6/neurintsurg-2021-SNIS.163}

Introduction/Purpose Blebs of intracranial aneurysms are clear bulges or outpouchings of the aneurysms wall. Since irregularly shaped aneurysms, i.e. aneurysms with lobulated shapes including one or multiple blebs are associated with a higher rupture risk, bleb occurrence is important for

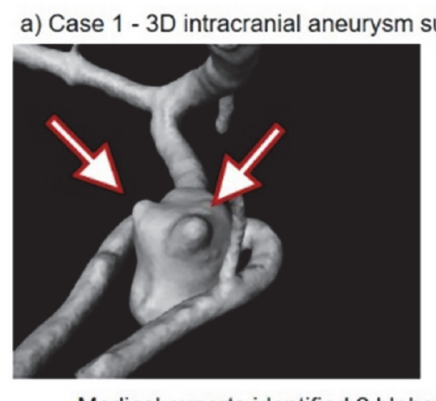

Medical experts identified 2 blebs

c) Case 2 - 3D intracranial aneurysm surface model

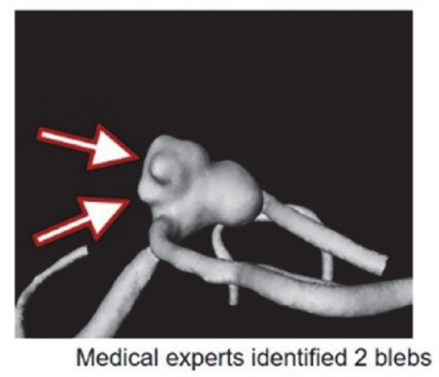

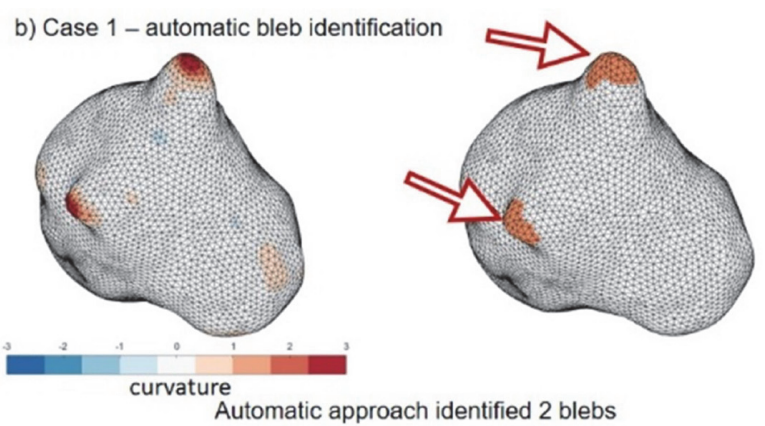

d) Case 2 - automatic bleb identification
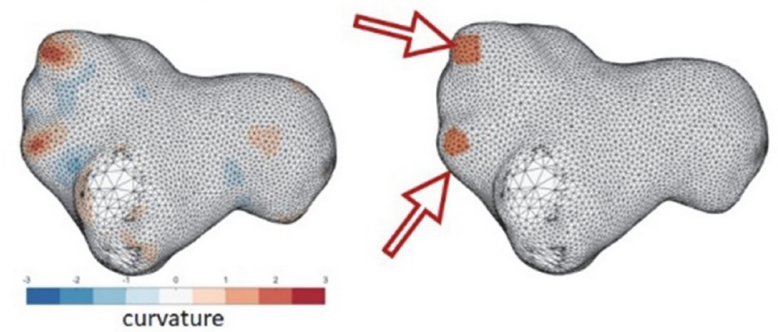

Automatic approach identified 2 blebs 